\title{
Andreas von Klewitz, Carl Chun, die Valdivia und die Entdeckung der Tiefsee
}

Berlin : Parthas Verlag Berlin, 2013, 215 p., 34,80€.

\section{Gaëlle Hallair}

\section{OpenEdition}

\section{Journals}

Édition électronique

URL : http://journals.openedition.org/ifha/8381

DOI : 10.4000/ifha.8381

ISSN : 2198-8943

Éditeur

IFRA - Institut franco-allemand (sciences historiques et sociales)

Référence électronique

Gaëlle Hallair, «Andreas von Klewitz, Carl Chun, die Valdivia und die Entdeckung der Tiefsee », Revue de I'IFHA [En ligne], Date de recension, mis en ligne le 01 janvier 2016, consulté le 22 septembre 2020. URL : http://journals.openedition.org/ifha/8381 ; DOI : https://doi.org/10.4000/ifha.8381

Ce document a été généré automatiquement le 22 septembre 2020

(CIFHA 


\section{Andreas von Klewitz, Carl Chun, die Valdivia und die Entdeckung der Tiefsee}

Berlin : Parthas Verlag Berlin, 2013, 215 p., 34,80€.

Gaëlle Hallair

1 Consacré à la première expédition scientifique allemande des grandes profondeurs océaniques, l'ouvrage relate le voyage de recherches entrepris en 1898 sous la direction du zoologue Carl Chun (1852-1914) à bord du navire Valdivia.

2 Renouant avec une ancienne tradition de rapports de voyage, l'auteur mêle à son récit de l'expédition des extraits du journal de C. Chun ainsi que des photographies et esquisses prises sur le terrain. Cet ouvrage relate donc le parcours professionnel de C. Chun tout en relevant de l'histoire des voyages et des expéditions scientifiques de la fin du XIX ${ }^{e}$ siècle, de l'histoire des sciences du terrain et de l'édition de sources. Cependant, dès l'introduction, l'auteur précise qu'il se place plutôt dans le champ de la littérature que dans celui de l'histoire des sciences.

C. Chun présente un parcours scientifique assez typique de la fin du XIX siècle, où les recherches de terrain sont le sésame indispensable pour une future carrière académique. A ce sujet, on renvoie le lecteur à un autre ouvrage de 2012 recensé dans la Revue de l'IFHA: Dag Henrichsen (dir.), Hans Schinz. Bruchstücke. Forschungsreisen in Deutsch-Südwestafrika. Briefe und Fotografien. Né près de Francfort/Main, à Höchst, C. Chun étudie la zoologie. A Leipzig, il soutient sa thèse en 1874 et son habilitation en 1878. Spécialiste des céphalopodes et du plancton, il entreprend plusieurs séjours de recherche sous forme d'expéditions océanographiques. Ces recherches de terrain lui assurent une brillante carrière académique : il devient professeur ordinaire (Maître de conférences) successivement à l'université de Königsberg en 1883 et de Breslau en 1891, puis professeur de zoologie à l'université de Leipzig. En 1908/1909, il devient recteur de l'université de Leipzig. Il rédige vingt-quatre tomes de rapports sur l'expédition du Valdivia. Parus de 1902 à 1940, ces derniers concernent quasi exclusivement la zoologie et le phytoplancton: Wissenschaftliche Ergebnisse der Deutschen Tiefsee-Expedition auf dem Dampfer "Valdivia" 1898-1899, réédités entre 2008 et 2013. En 1903 paraît également Aus den Tiefen des Weltmeeres. 
4 L'ouvrage de von Klewitz relève de l'histoire des voyages et des expéditions scientifiques caractéristiques de la fin du XIX siècle. Construit en 1886 en Angleterre, le Valdivia voyage 9 mois sur les mers pour l'expédition de C. Chun. Ses principales étapes de voyage sont: l'Atlantique Nord, les îles Canaries, l'Afrique occidentale (Cameroun, Congo), l'Atlantique Sud, Le Cap, l'île Bouvet, l'Antarctique et les îles Kerguelen, le sud de l'Océan indien, Sumatra, les Maldives, les Seychelles, l'Afrique de l'Est. L'expédition de C. Chun a pour objectif principal d'étudier les grands fonds océaniques sans cibler uniquement l'Antarctique. Peut-être est-ce la raison pour laquelle cette expédition a été moins publicisée que celle entreprise de 1901 à 1903 par Erich von Drygalski à bord du Gauss et souvent présentée comme la première expédition scientifique allemande vers l'Antarctique.

toutes les autres expéditions scientifiques réalisées sur terre comme sur mer celle du Valdivia est dédiée à la collecte de données scientifiques sur le terrain: des échantillons, des dessins et des photographies. L'équipe qui embarque avec le capitaine Adalbert Krech sous la direction du zoologue C. Chun comporte six autres scientifiques : un biologiste (Prof. W. Schimper), un océanographe (Dr. G. Schott), un chimiste (Dr. P. Schmidt), trois zoologues (Dr. C. Apstein, Dr. F. Braem, Dr. E. Vanhoeffen), un officier de navigation (W.Sachse), un médecin et bactériologiste (Dr. M. Bachmann), un photographe et dessinateur (F. Winter) et enfin un conservateur (R. Schmitt). Comme le montre la composition de l'équipe scientifique, les disciplines des sciences du terrain présentent des frontières très perméables. Les photographies et aquarelles réalisées au cours du périple expriment en outre un intérêt pour l'ethnologie (portraits d'Africains) et la géographie (paysages).

Concernant la structure de l'ouvrage, les 215 pages sont organisées comme dans un roman, en 21 chapitres, précédés d'une très (trop ?) brève introduction de l'auteur. L'entrée par la géographie l'emporte sur le séquençage chronologique puisque l'itinéraire suivi par le Valdivia sert de fil conducteur. L'illustration est riche et diversifiée, reflétant les archives et la collecte d'informations engrangées lors de ce périple sur les océans. Les sources iconographiques ainsi présentées le sont plus dans un souci d'illustration que dans celui de l'histoire de sciences et de l'édition d'archives.

7 Deux bémols: tout d'abord, si deux ou trois cartes régionales de l'époque sont reproduites comme sources, on regrette l'absence d'une carte synthétique. Une simple carte d'itinéraire, éventuellement avec les escales indiquées dans le récit, aurait permis d'élargir le registre des belles-lettres pour mieux souligner la grande porosité de l'époque entre les frontières disciplinaires, notamment entre la littérature de voyage et les sciences du terrain que sont la zoologie, l'océanographie, la géographie et l'ethnographie. Ensuite, on regrettera l'absence d'index de noms de personnes et de noms de lieux. Mais il est vrai que l'ouvrage de A. von Klewitz se tourne davantage vers le récit littéraire que vers l'histoire des sciences proprement dite. A ce propos, il est à rapprocher des travaux de Sophie Linon-Chipon et Daniela Vaj, notamment de leur ouvrage commun paru en 2006 : Relations savantes et discours scientifiques, Imago Mundi 12, Presses de l'Université Paris Sorbonne, Paris, 349 p.

8 Cet ouvrage reste néanmoins intéressant, notamment pour l'histoire des expéditions allemandes et pour la forme du récit qui incorpore astucieusement des extraits du journal de bord de C. Chun. Par ailleurs, on le mettra avec profit en rapport avec trois autres ouvrages, recensés dans la présente revue et traitant des sciences du terrain entre la fin du XIX ${ }^{\mathrm{e}}$ siècle et le début du XX ${ }^{\mathrm{e}}$ siècle : celui de Kurt Schmutzer, Der Liebe 
zur Naturgeschichte halber. Johann Natterers Reisen in Brasilien 1817-1836, (http:// ifha.revues.org/6514\#quotation) et un autre à paraître en 2015 : Martin Scharfe, Bilder aus den Alpen. Eine andere Geschichte des Bergsteigens, Wien, Köln, Weimar : Böhlau Verlag, 2013, $216 \mathrm{p}$.

INDEX

Index chronologique : Époque contemporaine

Thèmes : Sources, Histoire des sciences, Histoire des milieux naturels

\section{AUTEUR}

GAËLLE HALLAIR

CNRS-UMR 8504 Géographie-Cités, Paris 